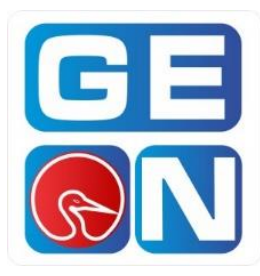

Revista GEON (Gestión, Organizaciones y Negocios.) ISSN: 2346-3910 en línea

revistageon@unillanos.edu.co

Universidad de los Llanos

Colombia

Vega, Alejandrai; Avila, Danae Duanaii.

Edu-entretenimiento y mercadotecnia de experiencia con niños como estrategia para la divulgación de ciencia y tecnología

Revista GEON, Vol. 5, No. 2, 2018

Pág. 50-67

Disponible en: https://doi.org/10.22579/23463910.4

${ }^{\mathrm{i}}$ https://orcid.org/0000-0002-2368-7939

ii https://orcid.org/0000-0003-2286-2843

Esta publicación se encuentra bajo licencia: Creative Commons

ReconocimientoNoComercialSinObraDerivada 4.0 Internacional

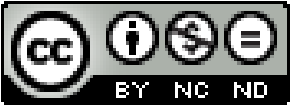

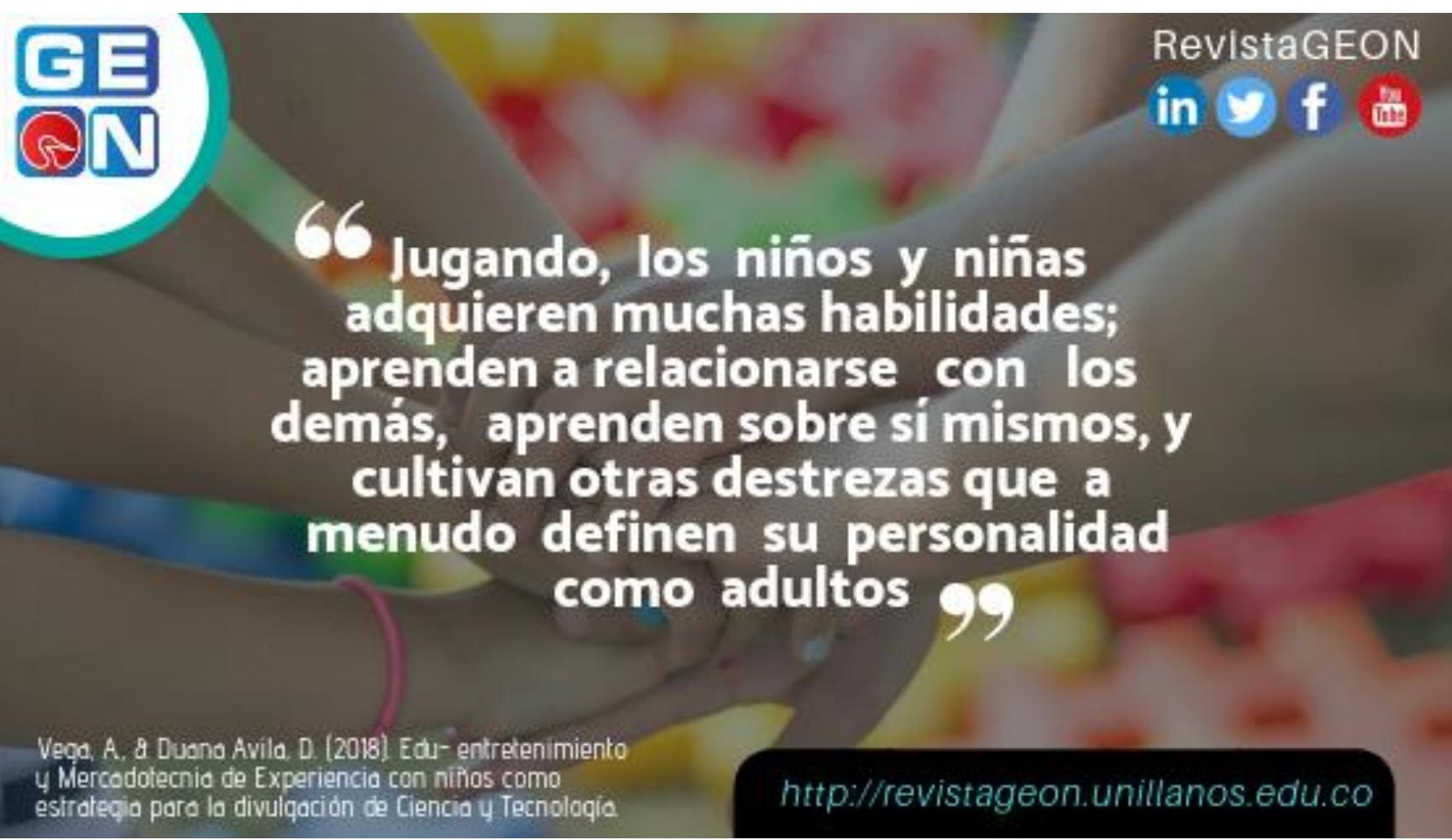




\title{
Edu-entretenimiento y Mercadotecnia de Experiencia con niños como estrategia para la divulgación de Ciencia y Tecnología
}

\section{Edu- Entertainment and Marketing of Experience with children as a strategy for the dissemination of Science and Technology}

\author{
Alejandra Vega Barrios \\ vbarrios@uaeh.edu.mx
}

Lic. Mercadotecnia y Doctora en proyectos línea en gestión empresarial, Instituto de Ciencias EconómicoAdministrativas (ICEA) - Universidad Autónoma del Estado de Hidalgo, México

\section{Danae Duana Ávila \\ duana@uaeh.edu.mx}

Doctor en ciencias económicas, Instituto de Ciencias Económico-Administrativas (ICEA) - Universidad Autónoma del Estado de Hidalgo, México

Revista GEON Vol. 5 No 2 julio-diciembre 2018

ISSN 2346-3910 en línea http://revistageon.unillanos.edu.co/index.php/geon/article/view/4 


\title{
Resumen
}

El mercado laboral actual, demanda a profesionales en un futuro con mayores herramientas de las ciencias duras, nuevas tecnologías, redes sociales, idiomas y con capacidad de coordinar y gestionar, por lo que es importante poner un nuevo contexto social a las generaciones que van incorporándose al ámbito escolar y que no vean "lo de siempre", sino nuevas formas de vivir a partir de espacios que muestren Ciencia y Tecnología, de manera divertida que los lleven a imaginarse dentro del entorno de una carrera universitaria.

Las tendencias generales muestran que el aprendizaje a partir de la experiencia mediante la integración de juegos, como el de ser profesionistas, utilizando técnicas lúdicas, las competencias y conocimientos cambian el contexto en el que se desenvuelve el individuo (niño) y con ello, podría cambiar las aspiraciones, ya que mediante la utilización del Edu- entretenimiento, y la mercadotecnia de experiencia es posible generar cambios de comportamiento aspiracional o apropiación social y realizar una mejor elección de carrera que les permita desarrollarse en este mundo por demás cambiante.

La presente propuesta pretende integrar la estrategia de comunicación para el cambio social denominada Edu- entretenimiento y la mercadotecnia de experiencia con la finalidad de contribuir en los principales aspectos de la divulgación de la ciencia y tecnología a partir del conocimiento de las profesiones ofertadas dentro de las universidades y con ello poder dar orientación a niños desde temprana edad en la elección de una carrera profesional.

Palabras clave: Edu- Entretenimiento, Mercadotecnia de Experiencia, Ciencia y Tecnología.

\begin{abstract}
The current labor market demands professionals in the future with hard sciences tools, new technologies, social networks, languages and with the capacity to coordinate and manage, so it is important to give a new social context to the generations that now in a school environment, that could shows Science and Technology, in a fun way that lead them to imagine in a university career.

General trends show that learning from experiencies through the integration of games, such as being as a professional, using play techniques, skills and knowledge change the context in which the child develops, could changes their aspirations, using Edutainment marketing experience it is
\end{abstract}


possible to generate changes in aspirational behavior or social appropriation and make a better choice that allows them to develop in this otherwise changing world.

The present proposal aims to integrate the communication strategy for social change called Edutainment and the marketing experience with the purpose of contributing to the main aspects of the dissemination of science and technology based on the knowledge of the professions offered within the Universities and thus give guidance to children in an early age in the choice of a professional career.

Keywords: Edu- Entertainment, Expertise Marketing, Science and Technology.

\section{Introducción}

El siguiente trabajo tiene como objeto estudiar la propuesta de un Modelo de negocio de Edu- entretenimiento y Mercadotecnia de Experiencia con niños como estrategia de divulgación de Ciencia y Tecnología en la ciudad de Pachuca, Hidalgo. México, con la finalidad de acercar a los estudiantes de primaria y secundaria a las ciencias básicas, generando mayor rendimiento académico en el nivel escolar actual y llevarlos a vislumbrar un mundo de ciencia y tecnología acorde a su edad cronológica y al aprendizaje significativo para introducirlos en el mundo de ciencias básicas.

Al conocer, los datos de rendimiento académico con respecto a las ciencias básicas (matemáticas y ciencias), los estudiantes denotan poco interés en el aprendizaje y esto recae en la deserción, por lo que por medio de un modelo de negocio con aspectos lúdicos (enseñanza y entretenimiento) muestren que las ciencias son importantes no solo para pasar un examen, sino para prepararse haciendo cosas comunes que se mueven con la aplicación de las matemáticas, realizando así Mercadotecnia de experiencia con la divulgación de Ciencia y Tecnología en un estado denominado "pobre".

De acuerdo con el Instituto Nacional de Estadística, Geografía e Informática (INEGI), en el apartado de Educación en Hidalgo (INEGI, 2010), En Hidalgo, el grado promedio de escolaridad de la población de 15 años y más es de 8.1 , lo que equivale a poco más del segundo año de secundaria.

De cada 100 personas de 15 años y más: $9 \%$ no tienen ningún grado de escolaridad; $59.2 \%$ tienen la educación básica terminada; $0.4 \%$ cuentan con una carrera técnica o comercial con primaria terminada; $18.0 \%$ finalizaron la educación media superior; $12.9 \%$ concluyeron la educación superior y $0.5 \%$ no especificado.

Lo más preocupante es que en el estado de Hidalgo, 10 de cada 100 personas de 15 años y más, no saben leer ni escribir y es por lo que se requiere ayudar a las familias y al municipio de 
Pachuca, Hidalgo. De acuerdo a las estadísticas de la Secretaria de Educación (SEP) el panorama general de la educación en Hidalgo para el ciclo 2015 - 2016 se muestra en la siguiente tabla (Secretaria de Educación Pública, 2017)

$\underline{\underline{\text { Tabla 1. Matricula del Sistema Educativo Hidalgo (Ciclo Escolar 2015 - 2016) }}}$

\begin{tabular}{|c|c|c|c|c|c|}
\hline \multirow[t]{2}{*}{ Nivel Educativo } & \multirow[b]{2}{*}{ Público } & \multirow[b]{2}{*}{ Privado } & \multicolumn{2}{|c|}{ Alumnos } & \multirow[t]{2}{*}{ Total, alumnos } \\
\hline & & & Hombres & Mujeres & \\
\hline Primaria & 3,001 & 253 & 181,269 & 174,527 & 355,796 \\
\hline \multirow{2}{*}{$\begin{array}{c}\text { Secundaria } \\
\text { Alcance Total }\end{array}$} & 1,138 & 149 & 86,977 & 85,440 & 172,417 \\
\hline & 4,139 & 402 & 268,246 & 259,967 & 528,213 \\
\hline
\end{tabular}

Fuente: Estadísticas del Sistema Educativo Hidalgo, ciclo escolar 2015 -2016

Sin embargo, se revisó el nivel de abandono de primaria a secundaria ya que a pesar de ser "obligatorio" el estudio de estos niveles, se tienen los siguientes datos.

\begin{tabular}{|c|c|c|c|c|}
\hline $\begin{array}{l}\text { Nivel } \\
\text { Educativ } \\
\text { o }\end{array}$ & $\begin{array}{l}\text { Abandon } \\
\mathrm{o} \\
\%\end{array}$ & $\begin{array}{l}\text { Reprobac } \\
\text { ión } \\
\%\end{array}$ & $\begin{array}{l}\text { Eficiencia } \\
\text { Terminal } \\
\%\end{array}$ & $\begin{array}{l}\text { Tasa de } \\
\text { Termina } \\
\text { ción } \\
\% \\
\end{array}$ \\
\hline Primaria & 0.0 & 0.4 & 103.4 & 108.9 \\
\hline $\begin{array}{l}\text { Secundari } \\
\text { a }\end{array}$ & 2.4 & 2.6 & 92.8 & 100.9 \\
\hline
\end{tabular}

Siendo que no es tan relevante el porcentaje de abandono en este momento, sin embargo, con este ritmo, el indicador para llegar al nivel educativo de licenciatura empieza a verse mermado, sobre todo en la intencionalidad de ciencias básicas.

Por otro lado en el mes de Septiembre de 2016, de acuerdo con la Agencia Informativa Conacyt se llevó a cabo el evento denominado "Primera Escuela de Ciencias Básicas" y las V Jornadas Académicas de Ciencias Básicas en el marco de su 40 aniversario, donde el objetivo fundamental fue el análisis y propuesta de las principales problemáticas que rodean la impartición de las asignaturas de ciencias básicas, desde el nivel básico hasta el posgrado, siendo de las acciones necesarias para poder tener mejores profesionistas en este campo. Dentro de este evento el Docente Jerónimo Gómez Rodríguez, asesor de bachillerato manifestó que la Escuela de Bachilleres ha identificado que la falta de afinidad de los jóvenes hacia las Ciencias Básicas se debe en gran medida, a los prejuicios familiares que predisponen a los estudiantes a no involucrarse en estas áreas de conocimiento, porque son complicadas, lo que limita la visión. Sin embargo, cuando se le invita a la participación de proyectos cambia la percepción (Agencia Informátiva Conacyt, 2016)

Por esto que existe evidencia que las mejores experiencias de aprendizaje han sido generadas mediante la integración de actividades significativas. 


\section{Revisión de la Literatura}

A partir de la literatura existente, se revisa que nuestro país al pertenecer al grupo de paises que producen y exportan materias primas e importa bienes basados en el conocimiento, lo que da pauta a preguntarse ¿ $\mathrm{Si}$ tenemos todos los recursos, porque compramos los productos terminados a otros paìses?, teniendo como respuesta que la generación de riqueza a partir de los recursos naturales que tiene México, es el costo de la ignorancia, ya que no se tienen suficientes ingenieros, tecnológos y cientificos (Contreras \& Flores, 2006).

Según Molano (2015), en México se eligen carreras poco redituables y con alto riesgo de desempleo, por lo que los padres deben ayudar a sus hijos a reflexionar acerca de sus fortalezas y debilidades, su capacidad de compromiso con una carrera corta o larga, su persistencia frente a las dificultades y tolerancia a la frustración. siendo esto el principal apoyo en su crecimiento.

En datos obtenidos del observatorio laboral, (STPS, 2016) se observa una saturación de personas preparadas para los temas administrativos, económicos, sociales por lo que se tiene que considerar que "La sociedad está cambiando y con ella la estructura del trabajo y las necesidades de las empresas".

No se ha logrado la vinculación a las caracteristicas culturales, sociales y económicas del mundo actual; ya que en las escuelas pareciera que las fuentes de conocimiento son las de siglos anteriores y no de nuevas herramientas para atraer a los niños a las necesidades del mercado de conocimiento.

El mercado demanda a profesionales en un futuro con mayores herramientas de las ciencias duras, nuevas tecnologías, redes sociales, idiomas y con capacidad de coordinar y gestionar, por lo que son los jóvenes quienes pueden incorporarse a la creación del conocimiento y de los bienes necesarios para el progreso de nuestra sociedad a través del estudio en carreras profesionales que tengan aspectos productivos como las ingenierías, las matemáticas, la física, la química y la biología, entre otras. (Contreras \& Flores, 2006).

María Soledad del Río, psicóloga clínica y vocacional de la Universidad de Chile, explica que mientras más joven, más difícil es decidir. "Esto, por la falta de conocimiento de las carreras y la inexperiencia en el mundo laboral, lo que los obliga a hacer un esfuerzo mayor de proyección en un ámbito para ellos desconocido".(Ministro de Educación, 2016).

En general, la ciencia y el conocimiento permiten mejorar las condiciones de vida de los seres humanos, y por ello que este trabajo pretende poder orientar mediante la divulgación de la Ciencia y Tecnología las distintas carreras que se ofertan en la Universidad Autónoma del Estado de Hidalgo para los jóvenes, con ello pudiesen imaginar su futuro profesional.

Es por ello que los beneficiarios de esta propuesta dentro de la sociedad son los niños de 6 a 14 años de la ciudad de Pachuca, Hidalgo, quienes gozaran de un 
lugar que los oriente y les muestre de forma lúdica la oferta educativa y proporcione apropiación social de la misma, siendo semillero de nuevas generaciones.

Edu - Entretenimiento. La comunicación y la tecnología digital, está alejando a los niños de los principales aspectos de sorpresa o investigación, al considerarlos nativos digitales estamos viviendo una revolución tecnológica que cambia nuestros hábitos de vida y afecta el entorno en el que nos desarrollamos, por lo que siguiendo a Ausubel (1983), en la Teoría del Aprendizaje Significativo, primeramente se revisó que se consideraba que el aprendizaje era sinónimo de cambio de conducta, porque dominaba la perspectiva conductista, sin embargo se vio que el aprendizaje humano va más allá de un cambio de conducta, ya que se produce un significado de la experiencia adquirida después del aprendizaje, lo que apertura la idea de enseñar a partir del entretenimiento.

Bandura (2000), habla de la teoría de aprendizaje social, como un enfoque centrado en la aplicación del aprendizaje por imitación a fin de obtener conductas de adaptación y éxito social, centrándose en los procesos de aprendizaje en la interacción entre el aprendiz y su entorno, por lo que conjuntando estos dos conceptos de aprendizaje e interacción podemos incorporar el concepto de "eduentretenimiento" que para (Marcos Américo, 2015) se refiere a la aplicación de espectáculos, shows, eventos, programas de radio y televisión con contenido educativo, afectando el cambio de comportamiento debido a la experiencia y al aprendizaje significativo.

García (2015), Menciona que existen distintos académicos del calibre Thomas Tufte, los cuales señalan que el eduentretenimiento es un sistema que beneficia en diversos ámbitos a los infantes; como en la conducta individual, planificación familiar, participación en comunidad, capacidad de identificación de problemas y proposición de soluciones.

Así mismo, el edu-entretenimiento cuenta con una gran variedad de juegos populares que permiten a niños y niñas pasar ratos agradables en familia.

Por otra parte, considerando la Teoría Socio Cultural donde Vygotsky (1985), establece que las interacciones favorecen el desarrollo de la ayuda activa o la construcción de andamiajes de un adulto o de alguien con más experiencia. La persona más experimentada puede dar consejos o pistas, hacer de modelo, hacer preguntas o enseñar estrategias, entre otras cosas, para que el niño pueda hacer aquello, que de entrada no sabría hacer solo.

Para que la promoción del desarrollo de las acciones autorreguladas e independientes del niño sea efectiva, es necesario que la ayuda que se ofrece esté dentro de la zona "de desarrollo próximo", una zona psicológica hipotética que representa la diferencia entre las cosas que el niño puede hacer a solas de las cosas ara las cuales necesita ayuda, aplicándose desde la educación básica hasta la media superior, considerando que llegue a ser inspirador y aspiracional para integrarse a una organización o institución. 
De acuerdo con Rodriguez (2007), se pretende con la teoría de acción razonada, y su implicación para el estudio de las actitudes, se pueda realizar una aportación hacia el estudio del comportamiento de los niños y adolescentes que sean invitados y evaluados, así como determinar la actitud sobre la publicidad boca a boca por identificar nuevos lugares a ser visitados y explotados como un lugar por conocer para jugar a ser el profesionista y el desarrollo de la actividad que genera el estudio de la Licenciatura o Ingeniería que se presente de forma lúdica, elaborando un índice de probabilidad de la intención hacia la conducta que relaciona la actitud con las creencias del individuo.

La teoría asevera que es posible predecir las conductas desde las actitudes, las intenciones conductuales y las creencias en este caso de la Institución de Educación Superior que abrirá sus puertas como parque temático para el conocimiento de las profesiones ofertadas y divulgación de ciencia y de esta forma definir la creencia como la probabilidad subjetiva de la relación entre el objeto de la creencia y algún otro objeto, concepto, valor o atributo.

Esto nos lleva a plantear la ventaja competitiva (Porter, 2007) entre sitios semejantes, la toma de acciones ofensivas o defensivas para crear una posición defendible en una industria, con la finalidad de hacer frente, con éxito, a las fuerzas competitivas y generar un Retorno sobre la inversión, que en este momento es oportuno por no tener ningún negocio similar en la ciudad de Pachuca, Hidalgo.
Lo visto de forma personal sobre este tipo de modelo de negocio es la denominada "Kidzania (Rosa, 2015)", donde se representa el mundo de los adultos imitando lo que ellos hacen, desde la tarea más simple como barrer o limpiar; o ingresar a trabajar con oficios definidos por las marcas participantes todo a escala infantil.

La diferencia estriba en que este parque temático solo fomenta oficios como: Reportero, Policía, Cocinero, Bombero, Electricista, Chofer, Jardinero, Cajero, Estudiante, Agente aduanal, Mensajero, Vendedor, Repartidos de periódico, Pintor y solamente se promueve como profesión la de médico, por lo que se pretende con este trabajo establecer profesiones no usuales en los juegos de los infantes, como son: Físico, Matemático, Químico, Ingeniero en Alimentos, Ingeniero Civil, Arquitecto, Ingeniero Industrial, Biólogo, Licenciado en Software, entre otras.

Mercadotecnia de Experiencia. De Siiri \& Jorma (2012) en su trabajo denominado "Marketing Theory: Experience Marketing and Experential Marketing" donde la revisión de la literatura determina que las experiencias se obtienen desde diversos puntos de vista, así como de las interpretaciones de los momentos, ya que cada vez las personas se encuentran buscando sentido a su vida, así como los principales aspectos de la felicidad, sensaciones, nuevas formas de llevar los valores fundamentales como lo han expresado.

Fortezza \& Pencarelli (2010), "La experiencia" en la comercialización suelen 
situarse entre lo genérico de "servicios". Sin embargo, la Experiencia en el Marketing es un nuevo enfoque para los negocios, comparado con el marketing tradicional, ya que al tener un enfoque innovador y creativo se llega más fácilmente a los consumidores.

La economía en la que viven los consumidores hoy en día se considera la economía de la experiencia. De las industrias que más están creciendo en este sentido es industria del entretenimiento, debido al valor de entretenimiento que los consumidores construyen experiencias en los contextos al utilizar la experiencia de marketing para atraer a más consumidores.

Para la correcta interpretación de la Mercadotecnia Experencial, iniciaremos con algunos antecedentes, entre los cuales se encuentra Philip Kotler, quién ha sido denominado "El padre de la Mercadotecnia", por sus distintas aportaciones a este campo.

De acuerdo con Kotler (2013), "Mercadotecnia se define como el proceso mediante el cual las empresas crean valor y generan fuertes relaciones con ellos para, en reciprocidad captar valor de los clientes" dando una experiencia única en la apropiación de un producto y/o servicio.

La Mercadotecnia de experiencia o las experiencias del consumidor, han dado origen a conceptos complejos, debido a que cada día el entorno es cambiante, así como las personas que se desarrollan en él ya que, al utilizar productos o servicios, se crean experiencias y ese mercado de las emociones se está volviendo muy importante. De acuerdo con Pine y Gilmore (1998) citado en (Moral Moral \&
Fernández Alles, 2012) afirma que "un individuo compra una experiencia y con ello paga para disfrutar una serie de sucesos memorables".

Los trabajos revisados sobre este tema tiene sus inicios en los 80 's, con las emociones como elemento clave en el comportamiento del consumidor, (Holbrook, 1982), aunque varios autores han retomado este concepto, nos referiremos a la cita que maneja Moral Moral \& Fernández Alles, (2012) con respecto a Pine y Gilmore (1998), donde se identifican cuatro tipos de experiencias del cliente en dos dimensiones.

El concepto multidimensional del marketing de la experiencia sugiere que los clientes pueden tener diferentes tipos de experiencias, según cita Barrios(2012), en el trabajo de Pine y Gilmore (1998). El éxito de una experiencia es que "un cliente la encuentre única, memorable $y$ sostenible en el tiempo" (p.12)., lo que nos muestra que los consumidores buscan que lo que se adquiera sea diferente para cada cliente, a pesar de ser el mismo articulo o servicios.

Esto se vuelve tangible cuando se conoce al comprador potencial, ya que de ello depende que la búsqueda o el hallazgo de la experiencia complemente lo que espera y lo que piensa para poder determinar el uso frecuente de la compra y de esta forma satisfacer la comunicación personal que en la actualidad se demanda.

\section{Divulgación de Ciencia y Tecnología.} A partir de la lectura denominada "Comunicación Educativa y Divulgación de la Tecnología y la Ciencia en y para el Sistema Nacional de Educación 
Tecnológica" (Ojeda \& Arevalo, 1988), en los últimos veinte años, la Secretaría de Educación Pública en México ha impulsado el desarrollo cultural, a partir de los sistemas de comunicación social.

No obstante, el tema de ciencia y la tecnología, no ha sido considerado al menos en este sector $y$ existe desconocimiento relativo a las carreras denominadas tecnológicas, aunque a partir de 1984, el Consejo del Sistema Nacional de Educación Tecnológica preparó un proyecto de comunicación social a corto mediano y largo plazo, considerando objetivos como:

Dar a conocer el Sistema Nacional de Educación Tecnológica, a través de una intensa promoción de sus escuelas, carreras, actividades y servicios de enseñanza e investigación e incorporar los medios electrónicos de comunicación y las nuevas tecnologías a las actividades y servicios del Sistema Nacional de Educación Tecnológica.

Con la principal intención de mejorar la calidad académica de enseñanza e investigación, apoyar la investigación científica y el desarrollo tecnológico en áreas estratégicas vinculadas a las necesidades del país.

Es en 1985 cuando a partir de un estudio analítico, se establecen las primeras formas de divulgación de Ciencia y Tecnología para niños, mediante el estudio denominado "Una Visión de la Divulgación de Tecnología y Ciencia para Niños", se ofrece un primer acercamiento técnico a la relación del niño con la ciencia y la tecnología; y sirve como base para producir mensajes impresos y audiovisuales adecuados para la población infantil.

Por lo que, si se identifica que una vocación se inculca desde la infancia, y de que los cambios de actitud hacia el conocimiento tecnológico y científico no se dan por sí solos, si no que se requiere de llevar y dirigir la Ciencia y la Tecnología al público infantil, para dar a conocer los principales aspectos que se manejan y que por desconocimiento no se han utilizado.

Los medios de comunicación se revelan hoy como una de las instancias de socialización juvenil por excelencia. Los informes de juventud realizados en la segunda parte de la década de los años noventa y los referentes a los primeros años del siglo XXI ponen de manifiesto cómo, a través de su tiempo y espacio de ocio, los jóvenes trabajan relaciones muy significativas con el entorno mediático. Su relación con los medios no sólo sirve para construir buena parte de su tiempo libre, o moldear su mundo de relaciones. Mar Chicharro Merayo (2014), Considerando que los más pequeños ya tienen contacto con los medios de comunicación, es importante identificar y llevarlos a conocer solo el resultado de la interacción con los medios, sino que los mismos han sido desarrollados con Ciencia y Tecnología que los llevaran a tener mejores oportunidades.

\section{Metodología y Análisis de los datos}

La metodología de Investigación que se empleó fue mixta, iniciando con Investigación Exploratoria con la finalidad de conocer a partir de la revisión de la literatura, 
mercadotecnia de experiencia y divulgación de ciencia y tecnología.

Por otra parte, se utilizó Investigación descriptiva con datos duros de clientes potenciales para esta, por lo propuesta que se tomará información del Instituto Nacional de Estadística, Geografía e Informática (INEGI), acorde a la pirámide poblacional y los quinquenios en los que se encuentra clasificada para la obtención cuantitativa.

Finalizando con la Investigación Correlacional, para establecer la relación que tiene el modelo de negocio con Edu entretenimiento y Mercadotecnia experiencial para la divulgación de ciencia y Tecnología.

\section{Hipótesis}

$\mathrm{H}_{1}$ : A mayor exposición de un espacio de modelo de negocio basado en Edu- entretenimiento y Mercadotecnia de Experiencia para divulgación de Ciencia y Tecnología, los niños de edades que oscilen entre 6 y 14 años tendrán mayor interés en carreras de Ciencias Básicas.

$\mathrm{H}_{0}$ : A mayor exposición de un espacio de modelo de negocio basado en Edu- entretenimiento y Mercadotecnia de Experiencia para divulgación de Ciencia y Tecnología, los niños de edades que oscilen entre 6 y 14 años no tendrán mayor interés en Ciencias Básicas.

Esta propuesta se sitúa bajo dos vertientes, por un lado en la Universidad Autónoma del Estado de Hidalgo (Universidad Pública) específicamente en la Ciudad del Conocimiento, en donde se tiene un circuito que es rodeado por áreas académicas dedicadas a las ciencias básicas.

Así mismo se consideraron dos escuelas (Primaria y Secundaria) dentro de un ámbito académico privado, siendo el escenario de estudio la Escuela Primaria y Secundaria Particular "Centro Cultural Simón Bolivar" de la Ciudad de Pachuca, Hidalgo y la Escuela Primaria y Secundaria "Centro Educativo Elise Freinet" durante el ciclo escolar 2016 2017 con la intención de realizar comparativos de apreciación.

Los participantes fueron una muestra intencional de estudiantes y padres de familia que se encuentran en los niveles escolares (Primaria y Secundaria), donde los sujetos de investigación serán cuestionados sobre el concepto de la propuesta y con ello se presente el espacio al que se requiere para el manejo de apropiación social.

Los estudiantes y padres de familia, tienen como característica esencial ser ajenos a la Universidad Autónoma del Estado de Hidalgo, por lo que el contexto en el que se desenvuelven en este momento radica a la Educación básica, por lo que tendrá un impacto esencial en el conocimiento de la Ciencia y la Tecnología a ser desarrollada.

La población total de niños de 6 a 14 años en la ciudad de Pachuca Hidalgo (INEGI, 2011) es de 65,577. Por lo que se calculó una muestra significativa con un error máximo aceptable del $5 \%$, con un porcentaje estimado de la muestra del $50 \%$ y un nivel deseado de confianza del $95 \%$, utilizando la fórmula 


$$
\mathrm{Z}^{2} *(\mathrm{p}) *(1-\mathrm{p})
$$

$$
c^{2}
$$

al sustituir valores en la formula, se obtiene como resultado que deben aplicarse 384 cuestionarios para tener una muestra representativa

$$
\frac{1.96^{2} *(.5) *(1-.5)}{0.05^{2}}=
$$

Los cuales fueron aplicados de forma proporcional en cada una de las escuelas (192 cuestionarios), y estos fueron distribuidos de manera uniforme entre los grados escolares como lo demuestra la siguiente tabla

Se consideró que la población total de ambas escuelas, siendo para el Instituto Cultural Simón Bolívar de 277 alumnos y por otra parte del Centro Escolar Elise Frainet $\quad 336$ alumnos y con ello se determinó que, por método no probabilístico de cuota, para garantizar la muestra establecida en el diseño del estudio.

\begin{tabular}{ll}
$\begin{array}{l}\text { Tabla 3. Distribución de cuestionarios por grado } \\
\text { escolar } \\
\text { Grado }\end{array}$ & $\begin{array}{l}\text { Número de } \\
\text { cuestionarios }\end{array}$ \\
Escolar & 12 \\
\hline \hline Primer grado primaria & 20 \\
Segundo grado primaria & 20 \\
Tercer grado primaria & 20 \\
Cuarto grado primaria & 20 \\
Quinto grado primaria & 25 \\
Sexto grado primaria & 25 \\
Primer año de secundaria & 25 \\
Segundo año de secundaria & 25 \\
Tercer año de secundaria & \\
\hline \hline
\end{tabular}

Fuente: Elaboración propia 2017
Los indicadores determinados en el cuestionario fueron extraídos a partir de instrumentos del libro "Marketing Scales Handbook Bearden, Netemeyer, \& Haws, (2011) los cuales han sido construidos, descritos, desarrollados, probados y validados en diferentes investigaciones, que se analizan y explican el objetivo de su uso.

A partir del instrumento y de la obtención de los datos, se observa en el análisis:

1. Primeramente estadísticas descriptivas, de la totalidad de los encuestados y diversificando las frecuencias desde el género y la edad, a los grupos que se han establecido para el estudio.

2. Consideración de los datos perdidos, promedios, desviación estándar, mínimo y máximos mediante tablas de contingencia.

3. Prueba "bootstrap" para determinar si existían diferencias significativas entre los grupos.,

Destacando que es información importante para entender que es lo que los niños buscan el juego y los padres están dispuestos a pagar por visitar un lugar de Edu - entretenimiento y Mercadotecnia de experiencia para conocer acerca de Ciencia y Tecnología, con la intención de poder acercar y orientar a los estudiantes de educación básica a un espacio lúdico que genere el interés por relacionarse con las ciencias básicas y con ello establecer nuevas formas de aprender y entender el uso de las mismas en la vida diaria. 


\section{Resultados y Conclusiones}

Se enviaron los cuestionarios a las dos instituciones participantes que fueron el Instituto Cultural Simón Bolívar y el Centro Escolar Elise Freinet, teniendo la siguiente distribución

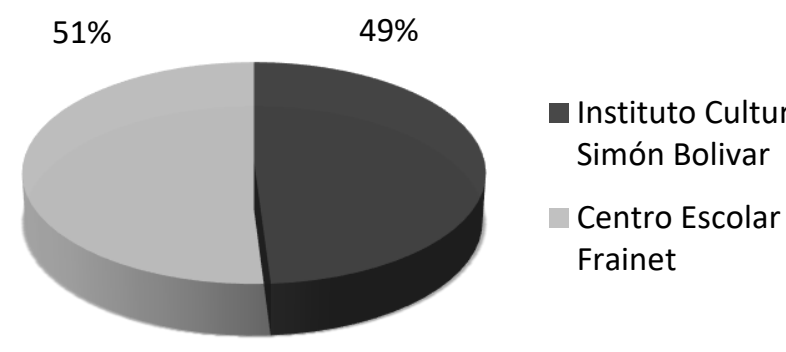

Gráfico 1. Distribución de Muestra por escuelas participantes

Fuente: Procesamiento datos SPSS, Elaboración propia 2017

De acuerdo con la distribución que se planteó se entrevistó 197 padres de familia del Centro Escola Elise Frainet que representa el $51 \%$ de los entrevistados y 187 del Instituto Cultural Simón Bolívar que representa el $49 \%$ de la muestra. (Gráfico 1)

Se aplicaron las mismas cantidades para Padres de Familia y Estudiantes.

Al determinar la frecuencia por género se encontró que contestaron la encuesta por parte de Padres de Familia, 188 personas de sexo Femenino representando el $48 \%$ de la muestra total y 196 de sexo masculino, que es el $52 \%$ de la muestra total (384).

En cuanto a los estudiantes de las dos instituciones se obtuvo que se entrevistaron 164 personas de sexo femenino representando el $42 \%$ de la muestra y 220 personas de sexo masculino, lo que representa el 58\% de la muestra total. (Gráfico 2)

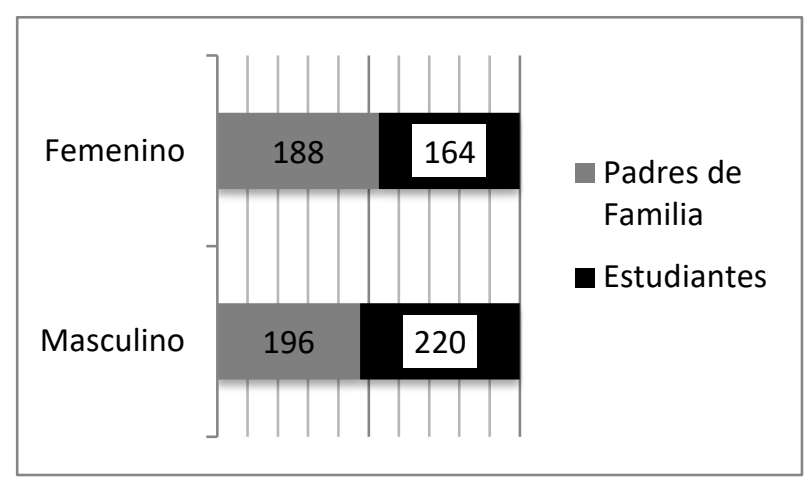

Gráfico 2. Distribución de Muestra por sexo y tipo de entrevistado

Fuente: Procesamiento datos SPSS, Elaboración propia 2017 escolar

Tabla 4. Frecuencia de cuestionarios por grado

\begin{tabular}{lllll}
\hline \hline $\begin{array}{l}\text { Nivel } \\
\text { Escolar }\end{array}$ & Frecuencia & Porcentaje & $\begin{array}{l}\text { Porcentaje } \\
\text { válido }\end{array}$ & $\begin{array}{l}\text { Porcentaje } \\
\text { acumulado }\end{array}$ \\
\hline \hline Primaria & 187 & 48.7 & 48.7 & 48.7 \\
Secundaria & 197 & 51.3 & 51.3 & 100.0 \\
Total & 384 & 100.0 & 100.0 & \\
\hline \hline
\end{tabular}
2017

Con esta información se analizó si los padres de familia consideran que la escuela a la que asisten sus hijos "utiliza metodología de estudio es adecuada para desempeñarse en una prueba" y si "hay una correspondencia entre la cantidad de tiempo que emplea mi hijo en estudiar y las notas que obtiene" encontrándose lo siguiente

Los padres de familia de primaria encuestados, el $37 \%$ están en desacuerdo en la metodología de estudio de su hijo para poder desempeñar una prueba, y el $40 \%$ si está De acuerdo, por lo que el $23 \%$ no puede determinar si está de acuerdo o no. 
En cuanto a la Secundaria los datos obtenidos de los 197 padres de familia encuestados refieren que el $41 \%$ está en desacuerdo, mientras que el $37 \%$ está de acuerdo, siendo que el $22 \%$ no puede determinar su posición.

Estos datos nos dan información sobre que lo que está aprendiendo los estudiantes, sus padres no consideran que tenga que ver con su desarrollo. (Tabla 5).

Tabla 5. Opinión de Padres de Familia con respecto a la Metodología de Estudio por grado escolar

Creo que la metodología de estudio de mi hijo es adecuada para posteriormente desempeñarse en una prueba

\begin{tabular}{lllll}
\hline \hline $\begin{array}{l}\text { Nivel } \\
\text { Escolar }\end{array}$ & $\begin{array}{l}\text { En } \\
\text { desacuerd }\end{array}$ & $\begin{array}{l}\text { Ni de acuerdo ni } \\
\text { en desacuerdo }\end{array}$ & $\begin{array}{l}\text { De } \\
\text { acuerdo }\end{array}$ & $\begin{array}{l}\text { Tota } \\
1\end{array}$ \\
Primaria & 69 & 42 & 75 & 186 \\
Secundaria & 82 & 41 & 74 & 197 \\
Total & 151 & 83 & 149 & 383 \\
\hline \hline
\end{tabular}

Fuente: Procesamiento datos SPSS, Padres de Familia.

Elaboración propia 2017.

Se les hizo la misma pregunta a los estudiantes de los niveles escolares de primaria y secundaria obteniendo que el $82 \%$ de estudiantes de nivel primaria consideran que su metodología de estudio es la adecuada y solo el $3 \%$ no lo considera. Por otra parte, los estudiantes de nivel secundaria están de acuerdo en un $83 \%$ y el $2 \%$ está en desacuerdo con la metodología de estudio que utiliza para la realización de un examen. (Tabla 6)

Tabla 6. Opinión de Estudiantes con respecto a la Metodología de Estudio por grado escolar

\begin{tabular}{lllll}
\hline \hline \multicolumn{5}{l}{$\begin{array}{l}\text { Crees que la metodología de estudio que utilizas es adecuada } \\
\text { para realizar un examen }\end{array}$} \\
\hline \hline Nivel & En & Ni de acuerdo & De & Total \\
Escolar & desacuerd & ni en & acuerd & \\
& o & desacuerdo & o & \\
Primaria & 6 & 26 & 154 & 186 \\
Secundaria & 4 & 28 & 165 & 197 \\
Total & 10 & 54 & 319 & 383 \\
\hline \hline \multicolumn{5}{r}{ Fuente: Procesamiento datos SPSS, Estudiantes por nivel } \\
escolar. Elaboración propia 2017
\end{tabular}

Así mismo se preguntó sobre la correspondencia que tiene la cantidad de tiempo de estudio con las notas obtenidas y de acuerdo a la apreciación de los padres de familia, en primaria el $40 \%$ está en desacuerdo con esta correspondencia, por el contrario, el 39\% está de acuerdo, sin embargo, el $19.8 \%$ no está de acuerdo ni en desacuerdo.

Los padres de familia de Secundaria consideraron en desacuerdo el $47 \%$, y de acuerdo con la relación entre el tiempo de estudio empleado y las notas obtenidas en un $36 \%$ lo que nos lleva a tener esta información para poder seguir con el planteamiento de Edu-entretenimiento. (Tabla 7).

Tabla 7. Correspondencia entre cantidad de tiempo de estudio y notas obtenidas (Padres de Familia) por grado escolar

\begin{tabular}{|c|c|c|c|c|}
\hline \multicolumn{5}{|c|}{$\begin{array}{l}\text { Creo que hay correspondencia entre la cantidad de tiempo que } \\
\text { emplea mi hijo en estudiar y las notas que obtiene }\end{array}$} \\
\hline $\begin{array}{l}\text { Nivel } \\
\text { Escolar }\end{array}$ & $\begin{array}{l}\text { En } \\
\text { desacuerd } \\
\text { o }\end{array}$ & $\begin{array}{l}\text { Ni de acuerdo } \\
\text { ni en } \\
\text { desacuerdo }\end{array}$ & $\begin{array}{l}\text { De } \\
\text { acuerd } \\
\text { o }\end{array}$ & Total \\
\hline Primaria & 76 & 37 & 73 & 186 \\
\hline Secundaria & 93 & 33 & 71 & 197 \\
\hline Total & 169 & 70 & 144 & 383 \\
\hline
\end{tabular}

Al realizar la pregunta a los estudiantes de primaria el $39 \%$ considera que está de acuerdo en la relación propuesta, sin embargo, el $40 \%$ no está de acuerdo.

Tabla 8. Correspondencia entre cantidad de tiempo de estudio y notas obtenidas (Estudiantes) por grado escolar

\begin{tabular}{lllll}
\hline \multicolumn{5}{l}{$\begin{array}{l}\text { Creo que hay correspondencia entre la cantidad de tiempo que } \\
\text { emplea mi hijo en estudiar y las notas que obtiene }\end{array}$} \\
\hline \hline Nivel & $\begin{array}{l}\text { En } \\
\text { desacuer }\end{array}$ & $\begin{array}{l}\text { Ni de } \\
\text { acuerdo ni en } \\
\text { Escolar }\end{array}$ & $\begin{array}{l}\text { De } \\
\text { acuer }\end{array}$ & Total \\
& do & 37 & 73 & 186 \\
Primaria & 76 & 33 & 71 & 197 \\
Secundaria & 93 & 70 & 144 & 383 \\
Total & 169 & \multicolumn{5}{c}{ Fuente: Procesamiento datos SPSS, Estudiantes por nivel escolar. } \\
\hline \hline \multicolumn{5}{r}{ Elaboración propia 2017. }
\end{tabular}


En cuanto a la secundaria, el $36 \%$ percibe de acuerdo la relación, mientras que $47 \%$ no está de acuerdo. (Tabla 8)

Tratando de entender, como aprenden los niños en la institución educativa (escuela) y la relación que los padres de familia mantienen con el conocimiento que adquieren sus hijos, se formularon preguntas para conocer si en tiempos libres o vacaciones los niños asisten a lugares de ciencia y tecnología.

Los hallazgos por nivel escolar y sexo de los padres de familia fueron los siguientes.

Tabla 9. Asiste a lugares de entretenimiento de Ciencia y Tecnología por grado escolar y por sexo del padre de familia

¿En general, le gusta llevar a su hijo a lugares de entretenimiento con juegos de ciencia y tecnología?

\begin{tabular}{lllll} 
Sexo & Nivel & & & Total \\
& Escolar & No & Si & \\
\hline \hline \multirow{3}{*}{ Masculino } & Primaria & 48 & 49 & 97 \\
& Secundaria & 51 & 48 & 99 \\
& Total & 99 & 97 & 196 \\
& Primaria & 50 & 39 & 89 \\
& Secundaria & 44 & 54 & 98 \\
& Total & 94 & 93 & 187 \\
\hline \hline
\end{tabular}

Fuente: Procesamiento datos SPSS, escolar. Elaboración propia 2017

Podemos observar que en realidad no es de interés llevar a los niños a lugares de entretenimiento de ciencia y tecnología, aunque al levantar la información nos percatamos que no tienen claro que son lugares de "Entretenimiento de Ciencia y Tecnología", por lo que al realizar la pregunta ¿Diría usted que conoce información acerca de lugares de juegos de Ciencia y Tecnología?, los resultados se definieron de la siguiente forma. (Tabla 10)

La tabla 10, nos muestra que en el $66.5 \%$ hay desconocimiento por parte de los padres de familia de lugares que ofrezcan entretenimiento de Ciencia y Tecnología al menos en este estudio en su localidad (Pachuca, Hidalgo), siendo que, si existen algunos lugares, que no se anuncian con estas características sin embargo se hacen funciones de Ciencia Básica, solamente el $33.4 \%$ conoce algunos lugares, lo cual nos ayuda para la propuesta de este estudio.

Tabla 10. Conoce información sobre lugares de Ciencia y Tecnología por grado escolar y sexo del padre de familia

\begin{tabular}{lllll}
\hline Sexo & Nivel & & & Total \\
& Escolar & No & Si & \\
\hline \hline \multirow{4}{*}{ Masculino } & Primaria & 70 & 27 & 97 \\
& Secundaria & 66 & 33 & 99 \\
& Total & 136 & 60 & 196 \\
Femenino & Primaria & 61 & 28 & 89 \\
& Secundaria & 58 & 40 & 98 \\
\multirow{2}{*}{ Primaria } & Total & 119 & 68 & 187 \\
& & 131 & 55 & 186 \\
& & 124 & 73 & 197 \\
\hline \hline
\end{tabular}

Fuente: Procesamiento datos SPSS, Conocimiento de padres de familia sobre información de lugares de Ciencia y Tecnología. Elaboración propia 2017.

Después de analizar los principales aspectos descriptivos, se realizó la búsqueda de datos perdidos, que se encontró que de la muestra de 384 encuestados de padres de familia, solo tenemos 1 cuestionario ya no efectuado y en el caso de las encuestas realizadas a los estudiantes pues posible obtener la muestra completa, ya que a pesar de ser un cuestionario autoadministrado se pidió a los encuestadores revisar que no faltará ningún dato.

Se muestra a continuación la prueba "bootstrap" que se utiliza frecuentemente para aproximar el sesgo o la varianza del análisis estadístico, para determinar alguna diferencia significativa y revisar los intervalos de confianza. 
Con base a estos resultados podemos determinar que la base de datos para estas variables presenta un dato perdido, de tal forma que las 383 observaciones son válidas.

La desviación estándar (típica) que se define como $\mathrm{S} / \sqrt{ } \mathrm{n}$, es de .5 y .8 sobre una media de 1.50 y 2.04 respectivamente que indican que la dispersión de los datos con respecto a la media es moderada, así como los valores máximos y mínimos de las variables. Por lo tanto, se considera que de las observaciones realizadas los lugares de entretenimiento de Ciencia y Tecnología no han sido considerados en los niveles escolares determinados Primaria y Secundaria por los padres de familia, por lo tanto, representan una oportunidad de negocio para que los estudiantes de estos niveles puedan aprender y conocer, dado que los alumnos no han sido orientados hacia las ciencias.

Siendo que los juegos desempeñan una función importante en el desarrollo de la infancia. Jugando, los niños y niñas adquieren muchas habilidades; aprenden a relacionarse con los demás, aprenden sobre sí mismos, y cultivan otras destrezas que a menudo definen su personalidad como adultos, se pudiese apoyar de esto para ofrecer un lugar en donde utilizando el juego se oriente a los estudiantes desde la Educación básica a la Ciencia y la Tecnología.

Tabla 11. Bootstrap por nivel Escolar, asistencia y Conocimiento de lugares de entretenimiento de Ciencia y Tecnología

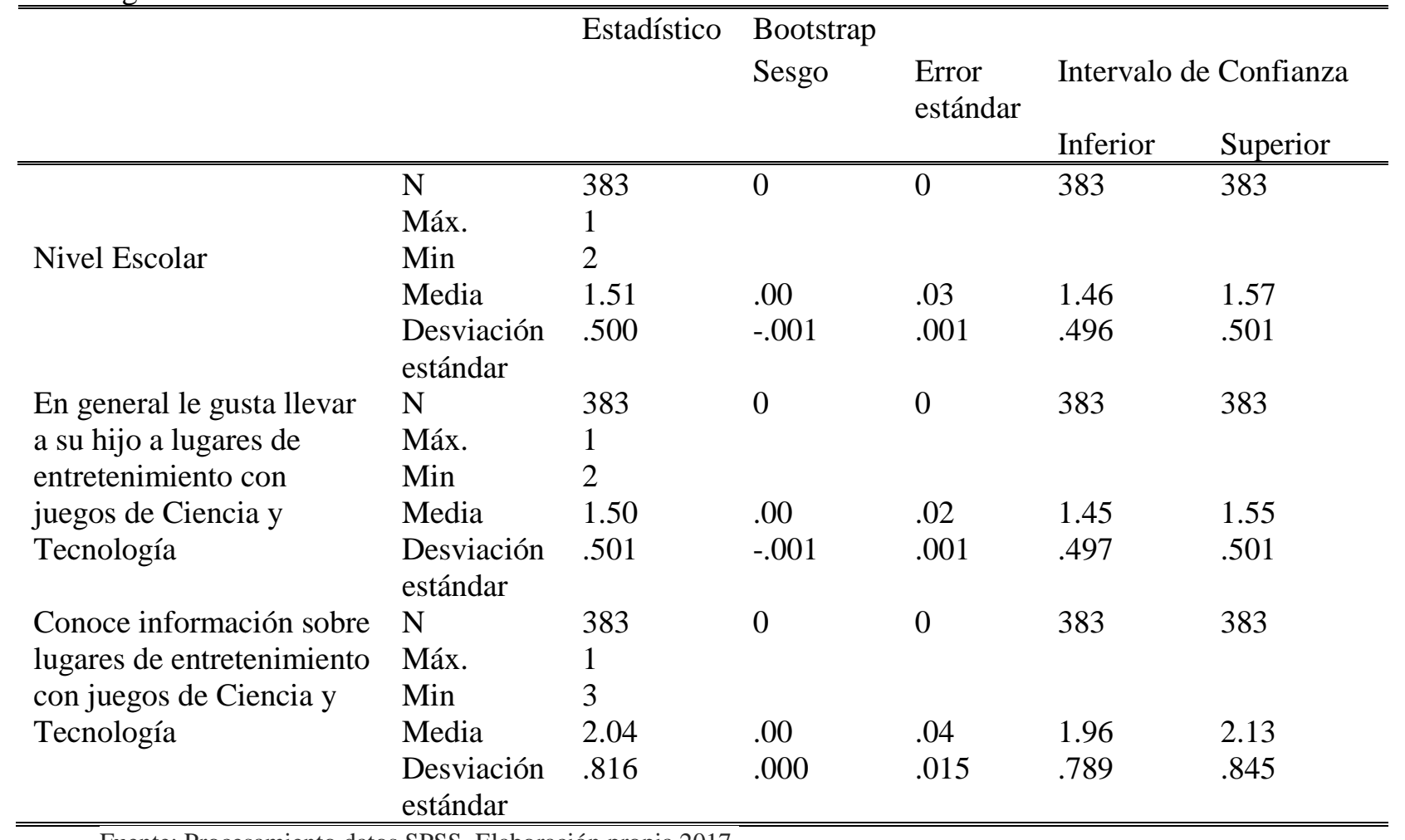

Fuente: Procesamiento datos SPSS. Elaboración propia 2017.

Siguiendo a Serrano, Sosa, \& Martínez (2015), en un modelo de negocio la parte más importante es la propuesta de valor es el factor que hace que un cliente se incline por una u otra empresa, su finalidad es solucionar un problema o 
satisfacer una necesidad del cliente. En este sentido, la propuesta de valor para solucionar constituye una serie de ventajas que se ofrecerán a quienes asistan a la Ciudad del Conocimiento como un parque temático en el que podrán encontrar lo que hacen los investigadores y conocer mediante la divulgación de la Ciencia y la Tecnología, lo que hacen las profesiones y como se desarrollan.

De tal suerte que se pueda realizar como parte del aprendizaje significativo un programa de reforzamiento de la enseñanza de las ciencias básicas, que se apoye del uso de las nuevas tecnologías de forma lúdica, considerando a su vez ciertos materiales impresos para propiciar el acercamiento de la familia, especialmente de los niños a este conocimiento, y que sirvan de instrumento a padres de familia y educadores.

Mediante videojuegos, realidad aumentada o realidad virtual, y desarrollo de películas con aspectos musicales o de animación que establezcan la oportunidad que viven actualmente los niños de 6 a 14 años, donde se puedan experimentar y manipular modelos que muestren fenómenos científicos y que complementen los contenidos de la educación básica, teniendo en cuenta los siguientes objetivos.

1) Identificar la Influencia de la motivación en el desempeño escolar del alumno de educación básica hacia las ciencias.

2) Conocer los antecedentes que influyen en el rendimiento escolar con respecto a ciencias básicas (matemáticas principalmente).
3) Identificar la influencia de la familia, amigos y entorno en relación a las Ciencias Básicas.

\section{Conclusiones}

Con ello se propone motivar a los niños de 6 a 14 años de la ciudad de Pachuca, Hidalgo mediante la visita a un parque temático científico a conocer las carreras que son impartidas en la máxima casa de estudios del Estado, la Universidad Autónoma del Estado de Hidalgo, haciendo vivencial (Marketing de experiencia) el conocimiento no solo de lo que se realiza, sino lo importante que es que entiendan que las ciencias básicas ayudan a tener mayores conocimientos y que nuestro país requiere de gente capacitada en estos temas para lograr mejorar la economía, además de entretener, educar y mantener la convivencia familiar, realizando apropiación social al desarrollo del estado en el que viven.

\section{Referencias}

Clark, T., Osterwalder, A., \& Pigneur, Y. (2012). Business Model You. En A. O. Tim Clark. Barcelona, España: Deusto.

Castillo, E. (Octubre de 2005). Escala Multidimensional SERVQUAL. Escala Multidimensional SERVQUAL RECOPILACIÓN. Chile.

CONACYT. (s.f.). Consejo Nacional de Ciencia y Tecnología. Recuperado el 28 de Noviembre de 2015, de http://www.conacyt.mx/

Coronado, J. (2007). Escalas de Medición. Paradigmas, 2, 104-125. 
Creative Research Systems . (27 de 12 de 2015). The survery system. Obtenido de http://www.surveysoftware.net/sscalce.htm

Agencia Informativa Conacyt. (11 de Septiembre de 2016). Analizan alternativas para la enseñanza de las Ciencias Básicas. http://www.conacytprensa.mx/index.php/socieda $\mathrm{d} /$ politica-cientifica/9801-analizan-alternativaspara-la-ensenanza-de-ciencias-basicas .

Asociación Mexicana de Agencias de Investigación y Opinión (AMAI). (Septiembre de 2014). Niveles Socio económicos. Recuperado el 17 de Junio de 2016, de AMAI: http://www.amai.org/

Ausubel, D. (1983). Teoría de Aprendizaje Significativo. México: Trillas.

Backhoff, E. (03 de Marzo de 2016). Estudiantes de bajo rendimiento en México. Opinión.

Bandura, A. (2000). Psicología Social. Obtenido de Adrián Triglia: http://psicologiaymente.net/social/bandurateoria-aprendizaje-cognitivo-social\#!

Barrios, M. (2012). Marketing de la Experiencia: Principales Conceptos y Características. Palermo Business Review (7), 67-89.

Bearden, W. O., Netemeyer, R. G., \& Haws, K. L. (2011). Handbook of Marketing Scales:Multi-item measures for marketing and consumer behavior research (Vol. 7). Thousand Oaks, California, United States of America: SAGE Publications, Inc.

Definición ABC. (2014). Retrieved 2016 йил 10-10 from Densidad de Población: http://www.definicionabc.com/geografia/densida d-de-poblacion.php

Fortezza, F., \& Pencarelli, T. (2010). Il marketing delle esperienze fra specificità e tendenze evolutive. Il caso Wish Days***. 9TH INTERNATIONAL CONGRESS "MARKETING TRENDS", (pp. 1-31). Venecia.
Global Industry Analysts. (Septiembre de 2015). Market Research.com. Recuperado el 30 de Marzo de 2017, de Global Industry Analysts: https://www.marketresearch.com/GlobalIndustry-Analysts-v1039/

García, F. (2015). Sistema educativo de carácter familiar que pretende integrar la comunicación y nuevas tecnologías como herramientas para el desarrollo. El Informador

Hamel, G. (2010). Del Conocimiento a la Creatividad. WOBI, Volumen 15.

Hernández-Sampierie, Roberto;. (2014). Definición del alcance de la Investigación. In R. Hernández Sampieri, C. Fernández Collado, \& M. d. Baptista Lucio, Metodología de la Investigación (6ta ed.). México, Distrito Federal, México: Mc Graw Hill.

Holbrook, М. (1982 йил Enero). The experiential aspects of consumption: Consumer fantasy, feelings and fun. Jornal of Customer Research, 9 (2), 132-140.

Horn, R., Wolff, L., \& Velez, E. (1991). Developing Educational assessment systems in Latin America: A review of issues and recent experience. Latin America and Caribbean Technical Department Regional Studies Program, Washington, D.C.

INEE. (2015). Panorama de la Educación en México. Indicadores del Sistema Educativo. México: Instituto Nacional para la evaluación de la Educación en México.

INEGI. (2010). EDUCACIÓN EN HIDALGO. Instituto Nacional de Estadística, Geografía e Informática. México: INEGI.

INEGI. (2011).

Panorama Sociodemográfico de Hidalgo. Ciudad de México: INEGI.

Kotler, P. (2013). Fundamentos de Mercadotecnia (Decimo primera ed.). Pearson Educación de México.

Marcos Américo, F. C. (2015). UN ACERCAMIENTO AL EDUENTRETENIMIENTO. QUESTIÓN , 1-5. 
Mar Chicharro Merayo. (2014). videojuegos y edu-entretenimiento. Revista de estudios de Juventud, 109-133.

Mathwick, Malhotra, \& Rigdon. (2001). Experiential Value Scale:EVS. In W. O. Bearden, R. G. Netemeyer, \& K. L. Haws, Handbook of Marketing Scales (Vol. 3, pp. 387-476). SAGE Publications, Inc.

Mendoza, M. (2000). Elección de Carrera Profesional: Visiones, Promesas y Desafíos. Ciudad Juárez, México: Universidad Autónoma de Ciudad Juárez.

Ministro de Educación. (10 de 06 de 2016). Descubre tu vocación. Obtenido de Mi futuro.cl: http://www.mifuturo.cl/index.php/un-asunto-devocacion-2/descubre-tu-vocacion

Molano, M. (19 de 08 de 2015). En México se eligen carreras poco redituables y con alto riesgo de desempleo. El Financiero.

Moral Moral, M., \& Fernández Alles, M. T. (2012). Nuevas Tendencias del Marketing: Marketing Experencial. Entelequia (14), 237-252.

Ojeda, G., \& Arévalo, J. (1988). COMUNICACION EDUCATIVA Y DIVULGACION DE LA TECNOLOGIA Y LA CIENCIA EN Y PARA EL SISTEMA NACIONAL DE EDUCACION TECNOLOGICA. Revista Telos (16).

Osterwalder, A. (2012). Tu modelo de negocio. En Iqbal Quadir, Tu modelo de Negocio. España: Deusto, Editorial Planeta.

Porter, M. (2007). Ventaja Competitiva. Copyright 2007 Online Executive Education.

Prim, A. (17 de Enero de 2015). Ejemplos de Modelos de Negocio. Recuperado el 12 de Junio de 2016, de Innokabi: innokabi.com/mimodelo-de-negocio-el-de-siempre/
Rodríguez, L. R. (2007). La Teoría de la Acción Razonada, implicaciones para el estudio de actitudes. INED, 66-77.

Rosa, A. d. (14 de Agosto de 2015). Kidzania vive un muy buen verano. El Economista.

Secretaria de Educación Pública. (Marzo de 2017). Indicadores y Pronósticos Educativos. Recuperado el 10 de Marzo de 2017, de Sistema Nacional de Información Estadística Educativa: http://www.snie.sep.gob.mx/indicadores_pronost icos.html

Serrano, J., Sosa, J., \& Martínez, P. (2015). EL TRABAJO COLABORATIVO COMO UNA ESTRATEGIA PARA LA FORMACIÓN DE LOS FUTUROS INGENIEROS. Anfei Digital, 1-9.

Siiri, S., \& Jorma, L. (2012). MARKETING THEORY: EXPERIENCE MARKETING AND EXPERIENTIAL MARKETING. 7th International Scientific Conference "Business and Management 2012" (pp. 480-487). LITHUANIA: Researchgate.

STPS. (10 de 06 de 2016). Observatorio Laboral. Obtenido de Secretaría del Trabajo y Previsión Social: http://www.observatoriolaboral.gob.mx/swb/es/o la/expectativas_laborales_futuro

Vygotsky, L. (1985). Instruccional design.org. Obtenido de Social Develop Theory: http://www.instructionaldesign.org/theories/soci al-development.html

Wang, M., Haertel, G., \& Walberg, H. (1993). Toward a Knowledge Base for School Learning. Review of Educational Research, 63 (3), 249-294. 